\title{
ISOLAMENTO DA YERSINIA PESTIS NOS FOCOS PESTOSOS DO NORDESTE DO BRASIL NO PERIODO DE 1966 A 1982
}

alzira Maria Paiva de alMeida (1), Darci Pascoal BRasil (1), Francisco Gomes de CARvalHo (2) e Célio Rodrigues de ALMEIDA (3)

\section{R E S U M O}

No período de 1966 a 1982 foram isoladas 861 cepas de Yersinia pestis sendo 471 originadas de material de roedores e outros pequenos mamíferos, 236 de lotes de pulgas, 2 de lotes de Ornithodorus e 152 de seres humanos dos focos pestosos do Nordeste do Brasil. Entre os roedores, a espécie que concorreu para o maior número de isolamentos foi o Zygodontomys lasiurus pixuna que, também, forneceu o maior número de lotes de pulgas naturalmente infectados, principalmente do gênero Polygenis. $O$ isolamento da Yersinia pestis de material proveniente de 13 Municípios do Estado de Pernambuco, 7 do Ceará, 3 da Paraíba, 1 do Piaui e 1 da Bahia, evidencia que o problema da peste nos focos brasileiros é bastante atual e merecedor de atenção. O maior número de cepas isoladas e de localidades afetadas, registradas no Estado de Pernambuco não significa maior incidência da peste no mesmo, mas é consequiência da pesquisa mais intensa da Yersinia pestis e da existência de laboratórios melhor preparados para o seu đ̆iagnóstico neste Estado.

\section{N T ROD U G A O}

A pesquisa contínua da Yersinia pestis nos focos pestosos do Nordeste do Brasil foi iniciada por ocasião de um programa de estudos sobre os fatores responsáveis pela focalização, epidemização e conservação da peste nestes focos e sobre o papel dos roedores silvestres e pulicídeos que ocorrem na região, no ciclo epidemiológico da peste (BALTAZARD ${ }^{5}$ ). Os resultados desses estudos permitiram introduzir modificações na metodologia da luta contra a peste.

A pesquisa do bacilo foi incorporada às operaçōes de vigilância da peste nos focos, visando-se a detecção precoce da atividade pestosa e acionamento imediato das medidas de controle, procurando-se dessa maneira evitar sua propagação na população humana. Durante os trabalhos de pesquisa e vigilância da peste desenvolvidos no períođo de 1966 a 1982 foram isoladas 861 cepas de Yersinia pestis. O presente artigo mostra a distribuição destas cepas quanto ao material de origem, área geográfica e ano de isolamento.

\section{MATERIAL E MÉTODOS}

A captura sistemática e ininterrupta de roedores e pulicideos para pesquisa da Yersinia pestis, foi iniciada em 1966 pelo Plano Piloto de Peste do CPqAM (FIOCRUZ) no Municipio de Exu - Pernambuco - Foco da Chapada do Araripe (BALTAZARD ${ }^{5}$ ). Nos demais focos foi progressivamente desenvolvida pela Campanha Contra a Peste da SUCAM/MS, a partir de 1975. Eventualmente o bacilo foi pesquisado em ou

(1) Centro de Pesquisas Aggeu Magalhães. Rua do Espinheiro, 106 - 50000 - Recife - Pernambuco - Brasil

(2) Laboratório de Peste do CPqAM. Exu - Pernambuco - Brasil

(3) Centro de Pesquisas Aggeu Magalhães. Rua do Espinheiro, 106 - 50000 - Recife - Pernambuco - Brasil (Bolsista do CNPq) 
ALMEIDA, A. M. P. de; BRASIL, D. P.; CARVAIHO, F. G. de \& ALMEIDA, C. R. de - Isolamento da Yersinia pestis nos focos pestosos do Nordeste do Brasil no período de 1963 a 1982. Rev. Inst. Med. trop. São Paulo 27:207-218, 1985.

tras fontes como pequenos marsupiais e insetos como ácaros e triatomíneos. As amostras de origem humana foram coletadas durante as investigações realizadas pela SUCAM dos casos notificados como suspeitos de peste (ALMEIDA \& col. $^{1)}$.

Dependendo da forma clínica da doença e do estado do paciente (BUTLER ${ }^{8}$ ) foi colhido suco bubonático, que constituiu a maior parte das amostras e/ou sangue. Nos casos de óbito amputava-se um dedo dos cadáveres para pesquisa do bacilo em uma das falanges (BAHMANYAR \& CAVANAUGH ${ }^{2}$ ). Foi também examinado esputo de 1 doente com suspeita de pneumonia pestosa primária.

O processamento dos roedores, insetos e material humano e o isolamento e a identificação do bacilo foram realizados segundo as técnicas preconizadas por BAHMANYAR \& CAVANAUGH ${ }^{2}$, BALTAZARD \& col. ${ }^{4}$ e KARIMI ${ }^{10}$, resultando no isolamento de 861 cepas. A clas sificação das cepas de Yersinia pestis não está baseada em características bioquímicas, antigênicas, fagotípicas etc., uma vez que este bacilo é bastante uniforme quanto à estes caracteres. Todas as cepas isoladas mesmo as originadas de material proveniente de outros Municípios, foram classificadas com a denominação P. EXU $(\mathrm{P}=$ peste $-\mathrm{EXU}=$ local onde a equipe que efetuou os isolamentos realizou a maior parte dos trabalhos), seguida do número de ordem dos isolamentos. A conservação das cepas foi realizada em tubos com camada alta de gelose, a $4^{\circ} \mathrm{C}$.

\section{RESULTADOS}

Os Quadros I, II, III, IV, V e VI mostram a distribuição das cepas de Yersinia pestis quanto à origem (roedores e outros pequenos mamíferos, pulicideos, ácaros e seres humanos) procedência (Focos/Municipios e Unidades da Federação) e ano em que foram isoladas. A distribuição do número de cepas de Yersinia pestis isoladas dos seres humanos por sexo e idade dos pacientes encontra-se no Quadro VII e a fonte do material de origem desses isolamentos é mostrada no Quadro VIII.

\section{DISCUSSĀO}

$O$ isolamento continuado da Yersinia pestis no período de 1966 a 1982 nos focos do Nor- deste do Brasil, evidencia a persistência desta infecção no nosso país, entretanto, os dados apresentados nos Quadros I a VI não permitem tirar conclusões sobre a flutuação da sua incidência uma vez que a colheita das amostras para estudo não foi realizada de maneira uniforme em todos os focos. Os isolamentos efetuados no período de 1966 a 1976 são na maioria provenientes do Município de Exu, Pernambuco, (Foco da Chapada do Araripe) em resultado à pesquisa ininterrupta do bacilo que vinha sendo realizada, até 1974 , apenas neste $\mathrm{Mu}$ nicípio. Nos outros Municípios, os isolamentos obtidos de 1966 a 1974 resultaram da coleta ocasional de amostras para exame, predominantemente de origem humana. A partir de 1977 quando a pesquisa da Yersinia pestis em material de roedores e pulicídeos já estava sendo sistematicamente realizada nos outros focos, os isolamentos foram originados principalmente de roedores do Foco do Agreste (Chapada da Borborema - PE, PB) dos Focos das Serras de Triunfo (PE, $\mathrm{PB}$ ), da Ibiapaba e Baturité (CE). Neste período o Foco da Chapada do Araripe (PE. PI, CE) mostrou-se menos ativo do que no precedente.

A colheita de amostras de material humano para pesquisa da Yersinia pestis só foi realizada de maneira sistemática nos Focos do Estado de Pernambuco e após 1975 no Foco da Serra da Ibiapaba - Ceará.

Em outros focos foi colhido somente material para diagnóstico sorológico dos doentes.

Por estes motivos não é possível comparar a distribuição da peste nos diversos focos. Entretanto, pode-se afirmar que no Município de Exu, onde esta infecção vem sendo pesquisada sem interrupção desde 1966, a mesma está quiescente há seis anos. A alternância de períodos de atividade com períodos de silêncio é característica universal dos focos de peste (OMS ${ }^{9}$ ). Em relação às Unidades da Federação, o maior número de isolamentos da Yersinia pestis foi obtido de material do Estado de Pernambuco, que também contou com o maior número de Municipios afetados. Isto não sig. nifica que a incidência da infecção seja maior neste do que nos outros Estados e sim uma consequiência da intensa pesquisa que vem sen. do ali realizada, além da existência de labora. 
Q U A D R O I

Distribuição das cepas de Yersinia pestis isoladas nos focos pestosos do Nordeste do Brasil no periodo de 1966 a 1982

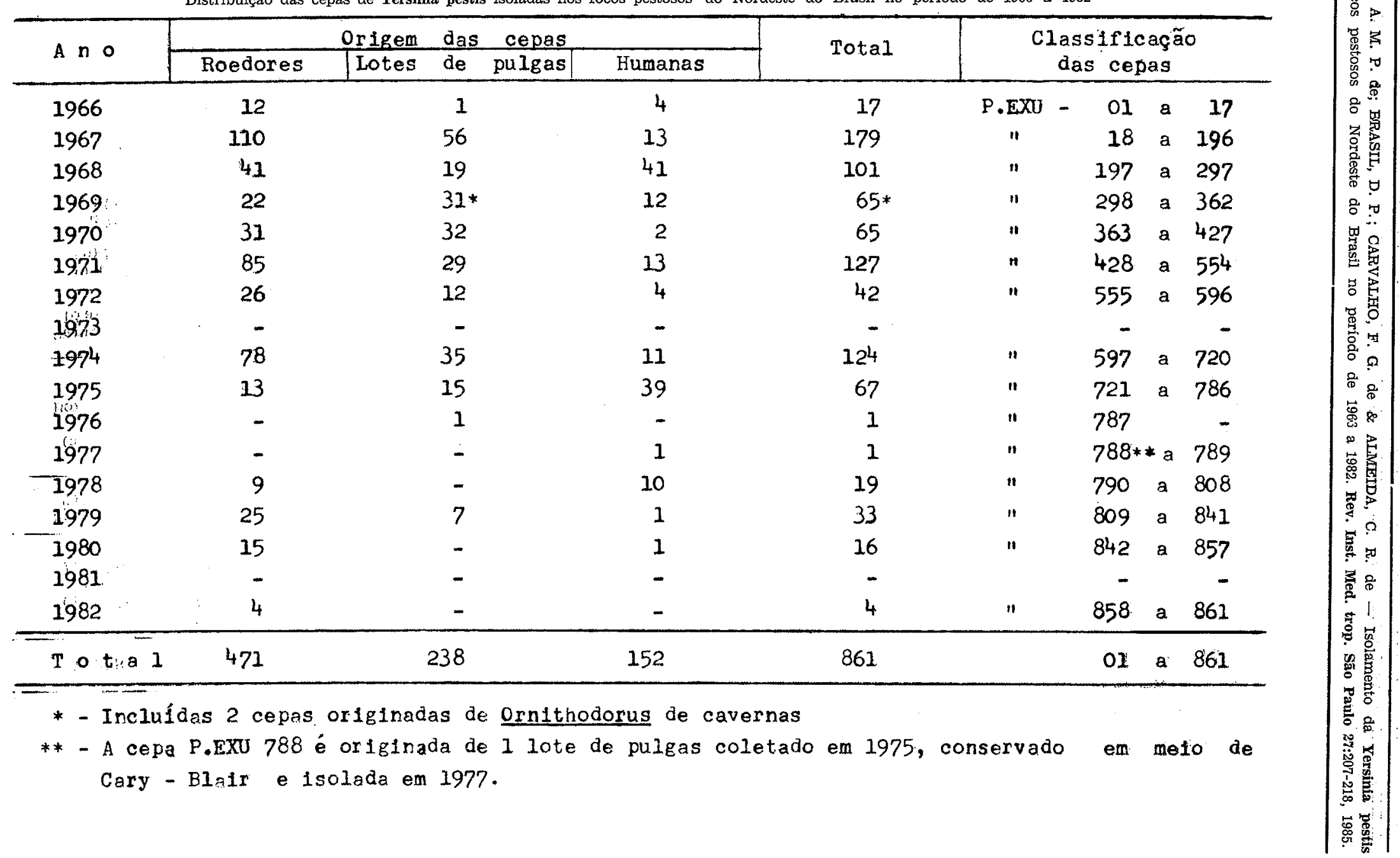


$Q$ U A D R O II

Distribuição das cepas de Yersinia pestis isoladas de roedores e outros pequenos mamíferos por espécie e local de origem, no periodo de 1966 a 1982

\begin{tabular}{|c|c|c|c|c|c|c|c|c|c|c|c|c|c|}
\hline \multirow{2}{*}{ Focos } & \multirow{2}{*}{ Locais } & \multicolumn{10}{|c|}{ Roedores e outros pequenos mamifer os } & \multirow{2}{*}{ Total } & \multirow{2}{*}{$\$$} \\
\hline & & Bra & $\operatorname{Rrf}$ & $\mathrm{Cc}$ & $\mathrm{Oe}$ & Os & Zlp & $\mathrm{Ta}$ & Gsw & Mdd* & S/inf. & & \\
\hline $\begin{array}{l}\text { Chapada } \\
\text { do } \\
\text { Araripe }\end{array}$ & $\begin{array}{l}\text { Exu - PE } \\
\text { Araripina-PE } \\
\text { Bodoco - PE } \\
\text { Ipubi - PE } \\
\text { Santana do Cariri - CE }\end{array}$ & $\begin{array}{r}17 \\
- \\
- \\
1\end{array}$ & $\begin{array}{r}12 \\
- \\
=\end{array}$ & $\begin{array}{l}1 \\
1 \\
- \\
-\end{array}$ & $\begin{array}{l}5 \\
- \\
- \\
-\end{array}$ & $\begin{array}{r}18 \\
\overline{1} \\
-\end{array}$ & $\begin{array}{r}334 \\
5 \\
7 \\
3 \\
-\end{array}$ & $\begin{array}{r}10 \\
2 \\
1 \\
-\end{array}$ & $\begin{array}{l}6 \\
- \\
- \\
-\end{array}$ & $\begin{array}{l}1 \\
- \\
1\end{array}$ & $\begin{array}{l}- \\
-\end{array}$ & $\begin{array}{r}404 \\
8 \\
9 \\
5 \\
1\end{array}$ & $\begin{array}{r}85,77 \\
1,70 \\
1,91 \\
1,06 \\
0,21\end{array}$ \\
\hline $\begin{array}{r}\text { Serra do } \\
\text { Triunfo }\end{array}$ & Triunfo- $\mathrm{PE}$ & 1 & - & - & - & 5 & 9 & - & - & - & - & 15 & 3,19 \\
\hline $\begin{array}{c}\text { Chapada } \\
\text { da } \\
\text { Borbor ema }\end{array}$ & $\begin{array}{l}\text { São Caetano - PE } \\
\text { Tacalmbó-PE } \\
\text { Macaparana - PE } \\
\text { Caetes - PE } \\
\text { Natuba - PB } \\
\text { Salgado de S.Fél1x - PB }\end{array}$ & $\begin{array}{l}- \\
- \\
-\end{array}$ & $\begin{array}{l}- \\
- \\
-\end{array}$ & $\begin{array}{l}- \\
- \\
-\end{array}$ & $\begin{array}{l}- \\
\dot{z} \\
- \\
-\end{array}$ & $\begin{array}{l}i \\
\overline{1} \\
\overline{1}\end{array}$ & $\begin{array}{l}9 \\
- \\
1 \\
2 \\
5 \\
1\end{array}$ & $\begin{array}{l}- \\
- \\
- \\
-\end{array}$ & $\begin{array}{l}- \\
- \\
- \\
- \\
-\end{array}$ & $\begin{array}{l}- \\
\overline{-} \\
-\end{array}$ & $\begin{array}{l}- \\
- \\
-\end{array}$ & $\begin{array}{l}9 \\
1 \\
2 \\
2 \\
6 \\
1\end{array}$ & $\begin{array}{l}1,91 \\
0,21 \\
0,43 \\
0,43 \\
1,27 \\
0,21\end{array}$ \\
\hline $\begin{array}{l}\text { Serra da } \\
\text { Ib1apaba }\end{array}$ & $\begin{array}{l}\text { Guaraciaba do Norte }-\mathrm{CE} \\
\text { Ipu - CE } \\
\text { Ipueiras - CE }\end{array}$ & 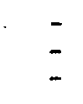 & $\begin{array}{l}- \\
-\end{array}$ & $\overline{-}$ & $\overline{-}$ & $\bar{z}$ & $\begin{array}{l}3 \\
1 \\
1\end{array}$ & 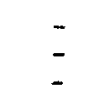 & $=$ & $\bar{z}$ & $\overline{1}$ & $\begin{array}{l}3 \\
2 \\
1\end{array}$ & $\begin{array}{l}0,64 \\
0,43 \\
0,21\end{array}$ \\
\hline $\begin{array}{l}\text { Serra do } \\
\text { Baturite }\end{array}$ & Aratuba - CE & - & - & 1 & - & - & - & - & - & - & - & 1 & 0,21 \\
\hline $\begin{array}{l}\text { Planalto } \\
\text { Oriental } \\
\end{array}$ & Serrinha - BA & - & - & - & - & - & - & - & - & - & 1 & 1 & 0,21 \\
\hline \multicolumn{2}{|r|}{ Tot a 1} & 19 & 12 & 3 & 6 & 27 & 381 & 13 & 6 & 2 & 2 & 471 & 100,00 \\
\hline \multicolumn{2}{|r|}{$\$$} & 4,03 & 2,55 & 0,64 & 1,27 & 5,73 & 80,89 & 2,76 & 1,27 & 0,43 & 0,43 & 100,00 & - \\
\hline $\begin{array}{l}\text { Rra - Hattus r } \\
\text { Rrf - Rattus r } \\
\text { Ce - Calomys }\end{array}$ & $\begin{array}{l}\text { rattus alexandrinus } \\
\text { rattus frugivorus } \\
\text { callosus }\end{array}$ & $\begin{array}{l}-O x \\
-O r \\
-z y\end{array}$ & $\begin{array}{l}\text { ryzomys } \\
\text { eyzomys } \\
\text { yodont }\end{array}$ & $\begin{array}{l}\text { el1u } \\
\text { subf } \\
\text { tomys }\end{array}$ & $\begin{array}{l}\text { rus } \\
\text { lavus } \\
\text { lasiuru }\end{array}$ & us Dix & xuna & $\begin{array}{l}\text { Ta - } \\
\text { Gsw - } \\
\text { Mdd - } \\
\text { S/Inf. }\end{array}$ & $\begin{array}{l}\text { richom } \\
\text { alea } s \\
\text { onodel } \\
\text { - Sem }\end{array}$ & $\begin{array}{l}\text { lys ape } \\
\text { pixil } \\
\text { phis d } \\
\text { Inform }\end{array}$ & $\begin{array}{l}\text { reoldes } \\
\text { wellsi } \\
\text {.domestio } \\
\text { açãa }\end{array}$ & ica (Mas & (plal) * \\
\hline
\end{tabular}


Q U A D R O III

Distribuição das cepas de Yersinia pestis isoladas de lotes de pulicídeos e de ácaros, por hospedeiros e local de origem, no período de 1966 a 1982

\begin{tabular}{|c|c|c|c|c|c|c|c|c|c|}
\hline \multirow{2}{*}{ Fuliclaeos e ácaros/Hospedelros } & \multicolumn{3}{|c|}{$\begin{array}{c}\text { Foco da Chapada } \\
\text { do Araripe }\end{array}$} & \multirow{2}{*}{\begin{tabular}{|c|}
$\begin{array}{c}\text { Foco da Se. } \\
\text { de . Triunfo }\end{array}$ \\
Triunfo \\
\end{tabular}} & \multirow{2}{*}{\begin{tabular}{|c|} 
Foco do \\
S.Caetano \\
\end{tabular}} & \multirow{2}{*}{$\begin{array}{l}\text { Agreste } \\
\text { Tacalmbó }\end{array}$} & \multirow{2}{*}{$\begin{array}{l}\text { Foco da Se: } \\
\text { da Ibiapaba } \\
\text { S. Benedito } \\
\end{array}$} & \multirow[t]{2}{*}{ Totai } & \multirow[t]{2}{*}{$\$$} \\
\hline & Araripina & Bodocó & Exu & & & & & & \\
\hline Polygenis /Rattus r.alexandrinus & - & - & 1 & - & - & - & - & 1 & 0,42 \\
\hline " Rattus r.frugivorus & - & - & 3 & - & - & - & - & 3 & 1,26 \\
\hline Calomys callosus & - & - & 1 & - & 1 & - & - & 2 & 0,84 \\
\hline Oryzonys eliurus & - & - & 4 & - & - & - & - & 4 & 1,68 \\
\hline OryzOmys subflavus & - & - & 6 & - & - & 1 & - & 7 & 2,95 \\
\hline zygodontomys 1. pixuna & - & 1 & 177 & 2 & 2 & 1 & 1 & $184_{4}$ & 77,31 \\
\hline Tr lchomys apereoides & 1 & 1 & 4 & - & - & - & - & 6 & 2,52 \\
\hline Galea splxil wellst & - & - & 1 & - & - & - & - & 1 & 0,42 \\
\hline Monodelphis d. domestica & - & - & 2 & - & - & - & - & 2 & 0,84 \\
\hline Sem Informação & - & - & 1 & - & - & - & - & 1 & 0,42 \\
\hline Xenopsylla/Rattus $r$ alexandrinus & - & - & 3 & - & - & - & - & 3 & 1,26 \\
\hline Rattus $r$.frugivorus & - & - & 3 & - & - & - & - & 3 & 1,26 \\
\hline Zygodontomys 1.p1xuna & - & - & 1 & - & - & - & - & 1 & 0,42 \\
\hline livre (casa) & - & - & 10 & - & - & - & - & 10 & 4,20 \\
\hline Porquinho da índia (casa) & - & - & 1 & - & - & - & - & 1 & 0,42 \\
\hline Ctenocephalldes/gato & - & - & 1 & - & - & - & - & 1 & 0,42 \\
\hline Pulex /livre (casa) & - & - & 6 & - & - & - & - & 6 & 2,52 \\
\hline Ornithodorus(acaro)/11vre (caverna) & - & - & 2 & - & - & - & - & 2 & 0,84 \\
\hline $\mathrm{T} \circ \mathrm{t} a \mathrm{I}$ & 1 & 2 & 227 & 2 & 3. & 2 & 1 & 238 & 100,00 \\
\hline$\$$ & 0,42 & 0,84 & 95,38 & 0,84 & 1,26 & 0,84 & 0,42 & 100,00 & - \\
\hline
\end{tabular}

7


Q U A D R O IV

Distribuição do número de cepas de Yersinia pestis isoladas de material humano, por ano e por local de origem, no período de 1966 a 1982

\begin{tabular}{|c|c|c|c|c|c|c|c|c|c|c|c|c|c|c|c|c|c|c|c|c|}
\hline Focos & Municipios / Ano & 1966 & 1967 & 1968 & 1969 & 1970 & 1971 & 1972 & 1973 & 1974 & +1975 & 1976 & 1977 & 71978 & 1979 & 1980 & 1981 & 1982 & Total & $\%$ \\
\hline $\begin{array}{l}\text { Chapada } \\
\text { do } \\
\text { Argripe }\end{array}$ & $\begin{array}{l}\text { Exu - PE } \\
\text { Araripina - PE } \\
\text { Bodoco - PE } \\
\text { Ouricuri - } \\
\text { S1mós - PI } \\
\text { Ioubi-PE } \\
\text { Granfto - PE }\end{array}$ & $\begin{array}{l}1 \\
- \\
- \\
- \\
-\end{array}$ & $\begin{array}{r}11 \\
= \\
= \\
=\end{array}$ & $\begin{array}{r}24 \\
6 \\
6 \\
- \\
- \\
-\end{array}$ & $\begin{array}{l}6 \\
- \\
1 \\
1 \\
- \\
2 \\
-\end{array}$ & $\begin{array}{l}1 \\
- \\
= \\
=\end{array}$ & $\begin{array}{l}1 \\
- \\
- \\
-\end{array}$ & $\begin{array}{l}= \\
= \\
= \\
=\end{array}$ & $\begin{array}{l}\bar{z} \\
\bar{z} \\
\overline{-}\end{array}$ & $\begin{array}{l}11 \\
\overline{-} \\
\overline{-} \\
-\end{array}$ & $\begin{array}{r}10 \\
22 \\
- \\
\overline{1} \\
5 \\
1\end{array}$ & $\begin{array}{l}- \\
\overline{-} \\
\overline{-} \\
\overline{-}\end{array}$ & & $\begin{array}{l}- \\
= \\
= \\
=\end{array}$ & $\begin{array}{l}\bar{z} \\
\bar{z} \\
\overline{-} \\
-\end{array}$ & $\begin{array}{l}\overline{-} \\
\overline{-} \\
\overline{1} \\
-\end{array}$ & $\begin{array}{l}= \\
\pm \\
= \\
=\end{array}$ & $\begin{array}{l}- \\
\\
- \\
-\end{array}$ & $\begin{array}{r}65 \\
28 \\
7 \\
1 \\
1 \\
8 \\
1\end{array}$ & $\begin{array}{r}42,76 \\
18,42 \\
4,60 \\
0,66 \\
0,66 \\
5,27 \\
0,66\end{array}$ \\
\hline Agreste & S/informaçáo- PE & - & 1 & - & - & - & - & - & - & & - & - & & - & - & - & - & - & 1 & 0,66 \\
\hline $\begin{array}{l}\text { Serra } \\
\text { da } \\
\text { Ibiapaba }\end{array}$ & $\begin{array}{l}\text { Ipu - CE } \\
\text { Ipue1ras - CE } \\
\text { G.do Norte - CE } \\
\text { S. Benedito-CE }\end{array}$ & $\begin{array}{l}- \\
-\end{array}$ & $\begin{array}{l}- \\
- \\
-\end{array}$ & $\begin{array}{l}\bar{z} \\
\overline{-}\end{array}$ & $\begin{array}{l}- \\
-\end{array}$ & $\begin{array}{l}- \\
-\end{array}$ & $\begin{array}{l}2 \\
1 \\
8 \\
1\end{array}$ & $\begin{array}{l}3 \\
1 \\
-\end{array}$ & $\begin{array}{l}- \\
-\end{array}$ & $\begin{array}{l}- \\
- \\
-\end{array}$ & $\begin{array}{l}- \\
-\end{array}$ & $\begin{array}{l}- \\
-\end{array}$ & & $\bar{z}$ & $\begin{array}{l}- \\
-\end{array}$ & $\begin{array}{l}- \\
- \\
-\end{array}$ & $\begin{array}{l}- \\
-\end{array}$ & $\begin{array}{l}- \\
- \\
-\end{array}$ & $\begin{array}{r}10 \\
1 \\
11 \\
2\end{array}$ & $\begin{array}{l}6,58 \\
0,66 \\
7,24 \\
1,31\end{array}$ \\
\hline $\begin{array}{c}\text { Serra } \\
\text { de } \\
\text { Baturité }\end{array}$ & $\begin{array}{l}\text { Aratuba - CE. } \\
\text { Capistrano - CE }\end{array}$ & $=$ & - & - & - & - & - & - & $=$ & $\overline{-}$ & - & - & & $\begin{array}{l}2 \\
1\end{array}$ & $\overline{-}$ & - & $=$ & - & $\begin{array}{l}2 \\
1\end{array}$ & $\frac{1,31}{0,66}$ \\
\hline $\mathrm{T}$ & o $t$ a 1 & 4 & 13 & 41 & 12 & 2 & 13 & 4 & - & 11 & 39 & - & 1 & 10 & 1 & 1 & - & & 152 & 100,00 \\
\hline & $\%$ & 2,63 & 8,55 & 26,97 & 7,90 & 1,31 & 8,55 & 2,63 & & 7,24 & $+25,66$ & & 0,6 & 66,58 & 0,66 & 0,66 & - & & 100,00 & - \\
\hline
\end{tabular}


Q U A D R O V

Distribuição do número de cepas de Yersinia pestis isoladas de material humano, de roedores e de lotes de pulicídeos por ano e local de origem, no periodo de 1966 a 1982

\begin{tabular}{|c|c|c|c|c|c|c|c|c|c|c|c|c|c|c|c|c|c|c|c|c|c|c|}
\hline Focos & Municipios/JF & 1966 & 1967 & 71968 & 1969 & 9197 & 1971 & 1972 & 1973 & 3197 & 974 & 1975 & 1976 & 61 & .977 & 1978 & 31979 & 1980 & 1981 & 1982 & 2 Total & $\not 8$ \\
\hline $\begin{array}{l}\text { Chapada } \\
\text { do } \\
\text { Araripe }\end{array}$ & $\begin{array}{l}\text { Exu - PE } \\
\text { Ararip̣ina - PE } \\
\text { Bodoco - PE } \\
\text { Ouricuri - PE } \\
\text { I pubí-PE } \\
\text { Granito - PE } \\
\text { Stna.Cariri - CE } \\
\text { Sinöes - PI }\end{array}$ & $\begin{array}{r}14 \\
= \\
= \\
= \\
=\end{array}$ & $\begin{array}{r}177 \\
- \\
- \\
- \\
-\end{array}$ & $\begin{array}{r}82 \\
7 \\
7 \\
- \\
= \\
- \\
-\end{array}$ & $\begin{array}{r}58 \\
\overline{1} \\
1 \\
2 \\
- \\
-\end{array}$ & $\frac{8}{2}$ & $\begin{array}{r}115 \\
= \\
= \\
= \\
=\end{array}$ & $\begin{array}{l}31 \\
\overline{6} \\
= \\
= \\
- \\
-\end{array}$ & $\begin{array}{l}\overline{-} \\
\overline{-} \\
\overline{-} \\
\overline{-}\end{array}$ & $=12$ & $\begin{array}{r}123 \\
= \\
= \\
= \\
\overline{1} \\
=\end{array}$ & $\begin{array}{r}31 \\
29 \\
- \\
\overline{5} \\
1 \\
\overline{1}\end{array}$ & $\begin{array}{l}1 \\
\\
2 \\
2 \\
- \\
-\end{array}$ & 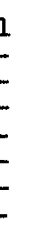 & $\begin{array}{l}\overline{-} \\
\bar{z} \\
\bar{z} \\
\overline{-}\end{array}$ & $\begin{array}{l} \\
\\
\end{array}$ & $\begin{array}{l}- \\
- \\
- \\
- \\
- \\
-\end{array}$ & $\begin{array}{l}\overline{1} \\
4 \\
\overline{6} \\
- \\
-\end{array}$ & $\begin{array}{l}\overline{-} \\
\overline{-} \\
\bar{z} \\
\overline{-}\end{array}$ & $\begin{array}{l}- \\
\overline{-} \\
-\end{array}$ & $\begin{array}{r}696 \\
=\quad 37 \\
=\quad 13 \\
=\quad 1 \\
-\quad 1 \\
1\end{array}$ & $\begin{array}{r}80,83 \\
4,30 \\
2,09 \\
0,12 \\
1,51 \\
0,12 \\
0,12 \\
0,12\end{array}$ \\
\hline $\begin{array}{l}\text { Chapada } \\
\text { da } \\
\text { Bor̈borema }\end{array}$ & $\begin{array}{l}\text { S/Informação - PE } \\
\text { Caetés - PE } \\
\text { Sao Caețano-PE } \\
\text { Tacalmbo - PE } \\
\text { Macaparana - PE } \\
\text { Natuba - PB } \\
\text { Salgado S.Fél1x-PB }\end{array}$ & $\begin{array}{l}- \\
- \\
-\end{array}$ & 1 & 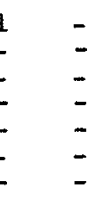 & $\begin{array}{l}- \\
\\
\end{array}$ & 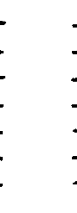 & $\begin{array}{l}\bar{z} \\
\bar{z} \\
\bar{z}\end{array}$ & $\begin{array}{l}- \\
- \\
= \\
=\end{array}$ & & 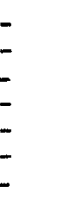 & $\begin{array}{l}\overline{-} \\
\overline{-} \\
\overline{-}\end{array}$ & 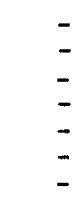 & $\begin{array}{l}- \\
\\
-\end{array}$ & 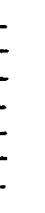 & $\begin{array}{l}\bar{z} \\
\bar{z} \\
\bar{z}\end{array}$ & $\begin{array}{l}- \\
\\
\\
-\end{array}$ & $\begin{array}{r}- \\
12 \\
3 \\
\overline{4} \\
-\end{array}$ & $\begin{array}{l}- \\
\overline{-} \\
\overline{2} \\
2 \\
-\end{array}$ & $\begin{array}{l}- \\
\overline{-} \\
- \\
-\end{array}$ & $\bar{i}$ & $\begin{array}{r}1 \\
2 \\
12 \\
3 \\
2 \\
6 \\
1\end{array}$ & $\begin{array}{l}0,12 \\
0,23 \\
1,39 \\
0,35 \\
0,23 \\
0,69 \\
0,12\end{array}$ \\
\hline $\begin{array}{c}\text { Serre } \\
\text { do } \\
\text { Triunfo }\end{array}$ & $\begin{array}{l}\text { Triunfo-PE } \\
\text { S.J.PeImonte - PB } \\
\text { Manaira - PB }\end{array}$ & $\begin{array}{l}2 \\
1 \\
-\end{array}$ & 1 & 5 & $\begin{array}{l}2 \\
1 \\
-\end{array}$ & 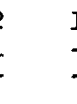 & $\overline{-}$ & $\overline{-}$ & & 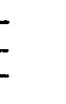 & $\begin{array}{l}- \\
-\end{array}$ & - & 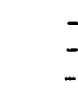 & & $\begin{array}{l}- \\
-\end{array}$ & $\begin{array}{l}6 \\
- \\
-\end{array}$ & $\frac{10}{1}$ & $\begin{array}{l}- \\
-\end{array}$ & $\overline{-}$ & & $\begin{array}{r}22 \\
7 \\
1\end{array}$ & $\begin{array}{l}2,55 \\
0,81 \\
0,12\end{array}$ \\
\hline $\begin{array}{c}\text { Serra } \\
\text { da } \\
\text { Iblapaba }\end{array}$ & $\begin{array}{l}\text { G.do Norte - CE } \\
\text { Ipu-CE } \\
\text { Ipueiras - CE } \\
\text { S.Benedito - CE }\end{array}$ & $\begin{array}{l}- \\
- \\
-\end{array}$ & - & 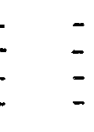 & $\overline{-}$ & 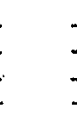 & $\begin{array}{l}8 \\
2 \\
1 \\
1\end{array}$ & $\frac{\frac{1}{3}}{1}$ & $\overline{-}$ & 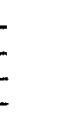 & $\begin{array}{l}- \\
-\end{array}$ & - & $\begin{array}{l}- \\
-\end{array}$ & & $\begin{array}{l}1 \\
- \\
-\end{array}$ & $\begin{array}{l}\frac{1}{6} \\
\frac{1}{1}\end{array}$ & $\begin{array}{l}2 \\
1 \\
- \\
-\end{array}$ & $\begin{array}{l}1 \\
- \\
-\end{array}$ & $\begin{array}{l}- \\
-\end{array}$ & $\overline{-}$ & $\begin{array}{r}14 \\
12 \\
2 \\
3\end{array}$ & $\begin{array}{l}1,62 \\
1,39 \\
0,23 \\
0,35\end{array}$ \\
\hline $\begin{array}{c}\text { Serra } \\
\text { de } \\
\text { Baturfté }\end{array}$ & $\begin{array}{l}\text { Aratuba - CE } \\
\text { Caplstrano - CE }\end{array}$ & - & & - & - & 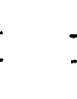 & - & - & & - & - & - & - & & - & $\begin{array}{l}3 \\
1\end{array}$ & $=$ & - & - & - & $\begin{array}{l}3 \\
1\end{array}$ & $\begin{array}{l}0,35 \\
0,12\end{array}$ \\
\hline $\begin{array}{l}\text { Planalto } \\
\text { Orlental } \\
\end{array}$ & Serrinha - BA & - & - & - & - & 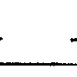 & - & - & - & - & - & - & - & & - & 1 & - & - & - & - & 1 & 0,12 \\
\hline & $\mathrm{T} 0 \mathrm{t}$ a 1 & 17 & 179 & 101 & 65 & 65 & 127 & $7 c$ & - & -12 & 24 & 67 & 1 & & 1 & 19 & 33 & 16 & - & 4 & \multirow[t]{2}{*}{8611} & 100,00 \\
\hline & $\%$ & 1,97 & & 911,73 & 7,55 & & 4,75 & 4,88 & & & & 7,78 & & & & & $3 ; 83$ & 1,86 & - & 0,46 & & - \\
\hline
\end{tabular}


Q U A D R O VI

Distribuição do número de cepas de Yersinia pestis isoladas de material humano, de roedores e de pulicideas por município e UF, no período de 1966 a 1982

\begin{tabular}{|c|c|c|c|c|c|}
\hline \multirow{2}{*}{$\mathrm{UF}$} & \multirow{2}{*}{ Municiptos } & \multicolumn{3}{|c|}{ Origem das cepas } & \multirow{2}{*}{ Total } \\
\hline & & \multirow[b]{2}{*}{$\begin{array}{c}\text { Roedores } \\
404 * \\
8 \\
9 \\
- \\
5 * \\
- \\
2 \\
9 \\
1 \\
2 \\
15 \\
- \\
-\end{array}$} & Lotes de pulgas & Humanas & \\
\hline $\mathrm{PE}$ & $\begin{array}{l}1 \text { - Exu } \\
2 \text { - Araripina } \\
3 \text { - Bodoco } \\
4 \text { - Ouricuri } \\
5 \text { - Ipubi } \\
6 \text { - Grantito } \\
7 \text { - Caetés } \\
8 \text { - São Caetano } \\
9 \text { - Tacalmbo } \\
10 \text { - Macaparana } \\
11 \text { - Triunfo } \\
12 \text { - S.Josédo Belmonte } \\
13 \text { - Sem informação }\end{array}$ & & $\begin{array}{c}227^{* *} \\
1 \\
2 \\
- \\
- \\
- \\
3 \\
2 \\
\overline{2} \\
- \\
-\end{array}$ & $\begin{array}{r}65 \\
28 \\
7 \\
1 \\
8 \\
1 \\
- \\
- \\
- \\
- \\
5 \\
7 \\
1\end{array}$ & $\begin{array}{r}696 \\
37 \\
18 \\
1 \\
13 \\
1 \\
2 \\
12 \\
3 \\
2 \\
22 \\
7 \\
1\end{array}$ \\
\hline CE & $\begin{array}{l}1 \text { - Santana do Cariri } \\
2 \text { - Guaraciaba do Norte } \\
3 \text { - Ipu } \\
4 \text { - Ipue1ras } \\
5 \text { - Sao Benedito } \\
6 \text { - Aratuba } \\
7 \text { - Capistrano }\end{array}$ & $\begin{array}{l}1 \\
3 \\
2 \\
1 \\
\overline{1} \\
-\end{array}$ & $\begin{array}{l}- \\
\bar{I} \\
-\end{array}$ & $\begin{array}{r}1 \overline{1} \\
10 \\
1 \\
2 \\
2 \\
1\end{array}$ & $\begin{array}{r}1 \\
14 \\
12 \\
2 \\
3 \\
3 \\
1\end{array}$ \\
\hline $\mathrm{PB}$ & $\begin{array}{l}1 \text { - Natuba } \\
2 \text { - Salgado de S.Fél1x } \\
3 \text { - Manaira }\end{array}$ & $\begin{array}{l}6 \\
1 \\
-\end{array}$ & $\overline{-}$ & $\overline{1}$ & $\begin{array}{l}6 \\
1 \\
1\end{array}$ \\
\hline $\mathrm{PI}$ & 1 - Simões & - & - & 1 & 1 \\
\hline $\mathrm{BA}$ & 1 - Serrinha & 1 & - & - & 1 \\
\hline & $\mathrm{T} \circ \mathrm{t} a \mathrm{l}$ & $471 *$ & $238 * *$ & 152 & 861 \\
\hline
\end{tabular}

* -2 cepas 1soladas de marsuplais (Monodelphis)

** - 2 cepas isoladas de ácaros (ornithodorus) 
Q U A D R O VII

Distribuição do número de cepas de Yersinia pestis isoladas de seres humanos por sexo e grupo etário dos pacientes, no período de 1966 a 1982

\begin{tabular}{|c|c|c|c|c|c|c|c|c|}
\hline \multirow{2}{*}{\multicolumn{3}{|c|}{$\begin{array}{l}\text { Idade } \\
\text { (Anos) }\end{array}$}} & \multicolumn{4}{|c|}{ S e $\times 0$} & \multirow{2}{*}{ Total } & \multirow{2}{*}{$\%$} \\
\hline & & & Femint no & $\%$ & Masculino & $\%$ & & \\
\hline 0 & - & 5 & 2 & 5,88 & 6 & 13,33 & 8 & 10,12 \\
\hline 6 & - & 10 & 7 & 20,59 & 10 & 22,22 & 17 & 21,52 \\
\hline 11 & - & 15 & 5 & 14,70 & 11 & 24,45 & 16 & 20,25 \\
\hline 16 & - & 20 & 4 & 11,77 & 6 & 13,33 & 10 & 12,66 \\
\hline 21 & - & 25 & 3 & 8,83 & 3 & 6,67 & 6 & 7,59 \\
\hline 26 & - & 30 & 2 & 5,88 & 1 & 2,22 & 3 & 3,80 \\
\hline 31 & - & 35 & 1 & 2,94 & 3 & 6,67 & 4 & 5,06 \\
\hline 36 & - & 40 & 2 & 5,88 & 1 & 2,22 & 3 & 3,80 \\
\hline 41 & - & 45 & 2 & 5,88 & - & - & 2 & 2,53 \\
\hline 46 & - & 50 & - & - & 2 & 4,45 & 2 & 2,53 \\
\hline 51 & - & 55 & 1 & 2,94 & - & - & 1 & 1,27 \\
\hline 56 & - & 60 & 3 & 8,83 & - & - & 3 & 3,80 \\
\hline 61 & - & 65 & 1 & 2,04 & 1 & 2,22 & 2 & 2,53 \\
\hline 66 & - & 70 & - & - & 1 & 2,22 & 1 & 1,27 \\
\hline$>$ & & 71 & 1 & 2,94 & - & - & 1 & $i 1,27$ \\
\hline Sub & $-t c$ & tal & 34 & - & 45 & - & 79 & - \\
\hline$s / 1$ & forn & açăo & 33 & - & 40 & - & 73 & - \\
\hline $\mathrm{T} 0$ & $t a$ & 1 & 67 & 44,08 & 85 & 55,92 & 152 & 100,00 \\
\hline
\end{tabular}


Q U A D R O VIII

Distribuição do número de cepas de Yersinia pestis originadas de material humano, quanto ao ano de isolamento e a fonte de material, no periodo de 1966 a 1982

\begin{tabular}{|c|c|c|c|c|c|c|c|}
\hline \multirow{2}{*}{$A$ no } & \multirow{2}{*}{$\begin{array}{c}\text { Ne de cepas } \\
\text { iscladas }\end{array}$} & \multicolumn{4}{|c|}{ Fonte de material } & \multirow{2}{*}{ Total } & \multirow{2}{*}{$\not \%$} \\
\hline & & Bubão & Sangue & Medula óssea & Esputo & & \\
\hline 1966 & 4 & 4 & - & - & - & 4 & 2,63 \\
\hline 1967 & 13 & 12 & 1 & - & - & 13 & 8,55 \\
\hline 1968 & 41 & 31 & 10 & - & - & 41 & 26,97 \\
\hline 1969 & 12 & 9 & 1 & 2 & - & 12 & 7,89 \\
\hline 1970 & 2 & - & 2 & - & - & 2 & 1,32 \\
\hline 1971 & 13 & 5 & 3 & 5 & - & 13 & 8,55 \\
\hline 1972 & 4 & 3 & 1 & - & - & 4 & 2,63 \\
\hline 1973 & - & - & - & - & - & - & - \\
\hline 1974 & 11 & 9 & 2 & - & - & 11 & 7,24 \\
\hline 1975 & 39 & 29 & 8 & 2 & - & 39 & 25,66 \\
\hline 1976 & - & - & - & - & - & - & - \\
\hline 1977 & 1 & - & 1 & - & - & 1 & 0,66 \\
\hline 1978 & 10 & 8 & 1 & - & 1 & 10 & 6,58 \\
\hline 1979 & 1 & 1 & - & - & - & 1 & 0,66 \\
\hline 1980 & 1 & - & - & 1 & - & 1 & 0,66 \\
\hline 1981 & - & - & - & - & - & - & - \\
\hline 1982 & - & - & - & - & - & - & - \\
\hline $\mathrm{T} \circ \mathrm{t} \quad \mathrm{a}$ & $1 \quad 152$ & 111 & 30 & 10 & 1 & 152 & 100,00 \\
\hline$\%$ & & 73,02 & 19,74 & 6,58 & 0,66 & 0,00 & - \\
\hline
\end{tabular}

|


ALMEIDA, A. M. P. de; BRASIL, D. P.; CARVALHO, F. G. de \& ALMEIDA, C. R. de - Isolamento da Yersinia pestis nos focos pestosos do Nordeste do Brasil no período de 196j a 1982. Rev. Inst. Međ̃. trop. São Paulo 27:207-218, 1985.

tórios melhor preparados para o seu diagnóstico.

Entre os roedores e pulicídeos, os mais altos percentuais de isolamento foram obtidos da espécie Zygodontomys lasiurus pixuna e suas pulgas Polygenis sp. em todos os focos.

Zygodontomys e Polygenis são considerados responsáveis pela amplificação do processo epizoótico nos focos do Nordeste do Brasil (BALTAZARD ${ }^{6}$ ).

$O$ isolamento da Yersinia pestis de pulgas "livres", dos gêneros Xenopsylla e Pulex, no piso das casas do homem do campo, reflete a im. portância destas espécies na origem dos casos humanos de peste. Por outro lado o encontro do bacilo da peste em pulgas de gato e porquinho da india mostra que os animais domésticos, também, podem estar envolvidos no cicio da infecção no nosso meio. Animais de estimação, principalmente cães e gatos são considerados importantes elos na transmissão da peste humana nos focos dos EEUU (BARNES 7). O encontro da Yersinia pestis em outras fontes como o pequeno marsupial Monodelphis domestica domestica e ácaros do gênero Ornithodo. rus significa que durante as grandes epizootias de peste dos roedores, outras espécies também podem ser atingidas $\mathrm{e}$ as chances do homem se infectar são grandemente ampliadas.

Pela distribuição da origem dos isolamentos, em relação à idade e sexo dos pacientes, verifica-se que a infecção atinge a todos os grupos etários de ambos os sexos com predominância maior nos indivíduos de 0 a 20 anos e principalmente no sexo masculino. Estas diferenças podem estar relacionadas à costumes regionais, indumentária utilizada e atividades exercidas (BUTLER 8 ). Quanto à fonte de material dos pacientes o maior número de isolamentos foi obtido de suco bubonático evidentemente por haver sido a principal fonte das amostras de origem humana examinadas. Embora não tenha sido realizada hemocultura de todos os pacientes, o encontro da Yersinia pes. tis em grande número de amostras de sangue de doentes que também tinham bubão infectado por este bacilo, evidencia que a forma bubosepticêmica da peste não é rara nos focos brasileiros. Esta forma clínica da doença, pode resultar na infecção das pulgas humanas, Pulex irritans, aumentando as chances da transmissão inter-humana através das picadas destas pulgas. BALTAZARD ${ }^{3}$ observou que este tipo de transmissão é responsável pelos surtos familiares de peste que ocorrem ocasionalmente após um caso fatal na residência.

A ocorrência de outra forma grave da doença, a peste pneumônica primária, de alto potencial de disseminação foi constatada pelo isolamento do bacilo pestoso no esputo de um paciente.

\section{SUMIMARY}

Isolation of Yersinia pestis in plague foci of Northeast Brazil from 1966 to 1982

From 1966 to 1982, 861 strains of Yersinia pestis were isolated from plague foci in Northeastern Brazil. The source of these isolates were: 471 rodents, 236 pools of fleas, 2 pools of Ornithodorus and 152 humans beings.

Zygodontomys lasiurus pixuna was the rodent specie that produced mostly of the isolates as well as mostly of the infected pools of fleas mainly of the Polygenis species.

The infected samples were originated from municipalities of Pernambuco (13) Ceará (7) Paraíba (3) Piauí (1) and Bahia (1) States. This geographic distribution shows that plague remains a health problem in Brazil and deserves attention.

The bigger numbers of isolates and affected areas in Pernambuco State does not necessarily indicates a higher infection incidence in such State but results from a more intense surveillance and skilled Laboratories for plague diagnosis.

\section{REFERENGIAS BIBLIOGRAFICAS}

1. ALMEIDA, C. R.; ALMEIDA, A. R.; VIEIRA, J. B.; GUIDA, U. \& BUTLER, T. - Plague in Brazil during two years of bacteriological and serological surveillance. Bull. Wld. Hlth. Org. 59: 591-597, 1981.

2. BahmaNyaR, M. \& CaVanaUGH, D. C. - Plague Manual. WHO, Geneva, 1976.

3. BALTAZARD, M. - Nouvelles donnees sur la transmission interhumaine de la peste. Bull. Acad. Nat. Méd. 143: 517-522, 1959.

4. BALTAZARD, M.; DAVIS, D. H. S.; DEVIGNAT, R.; GIRARD, G.; GOHAR, M. A.; KARTMAN, L.; MEYER, 
ALMEIDA, A. M. P. de; ERASIL, D. P.; CARVALHO, F. G. de \& ALIMEIDA, C. R. de - Isolamento da Yersinia pestis nos focos pestosos do Nordeste do Brasil no periodo de 1963 a 1982. Rev. Inst. Med. trop. São Paulo 27:207-218, 1985.

K. F.; PARKER, M. T.; POLLITZER, R.; PRINCE, F. M.; QUAN, S. F. \& WAGLE, P. - Recommended laboratory methods for the diagnosis of plague. Bull. Wld. Hlth. Org. 14: 457-509, 1956.

5. BALTAZARD, M. - Viagem de estudo ao Brasil para a organização de um projeto de pesquisas sobre a peste Rev. Bras. Malariol. Doenças Trop. 20: 335-366, 1968.

6. BALTAZARD, M. - Pesquisas sobre a peste no Brasil. Rev. Bras. Malariol. Doenças Trop. 20: 371-389, 1968.

7. BARNES, A. M. - Surveillance and control of bubonic plague in the United States. Symp. Zool. Soc. Lond. 50: 237-270, 1982.
8. BUTLER, T. - Plague and other Yersinia infections. New York, Plenum Medical Book Company, 1983.

9. CRONICA DE LA OMS - Vigilancia y lucha contra la peste. OMS, Ginebra, 34: 152-156, 1980.

10. KARIMI, $Y$. - Diagnostic rapide de l'infection pesteuse au laboratoire. Bull. Soc. Path. Exot. 71: 45-48, 1978.

Recebido para publicação em 28/5/1984. 